\title{
Phosphorus magnetic resonance spectroscopy in malformations of cortical development
}

\author{
Espectroscopia de fósforo por ressonância magnética em malformações do \\ desenvolvimento cortical
}

Celi Santos Andrade

\author{
Doutorado (Radiologia) na Faculdade de Medicina da Universidade de São Paulo (USP), 2011, São Paulo SP, Brazil. \\ Orientadora: Professora Doutora Claudia da Costa Leite \\ Correspondence: Celi Santos Andrade; Avenida Doutor Altino Arantes 370 / apto. 32; 04042-002 São Paulo SP - Brasil; E-mail: celis.andrade@hotmail.com \\ Support: The research was financed by FAPESP (CInAPCe project 05/56464-9). Dr. Celi Santos Andrade is supported by FAPESP (Grant 2012/00398-1).
}

\begin{abstract}
Introduction: Malformations of cortical development (MCD) result from disruptions in the dynamic process of cerebral corticogenesis and are important causes of epilepsy, motor deficits and cognitive impairment. Objectives: The aim of this study was to evaluate phospholipids metabolism in vivo in a series of patients with epilepsy and MCD. Methods: Thirty-seven patients with MCD and 31 control subjects were studied using three-dimensional phosphorus magnetic resonance spectroscopy ( ${ }^{3} \mathrm{P}-\mathrm{MRS}$ ) at a 3.0 T scanner. Quantification methods were applied to the following resonances: phosphoethanolamine (PE), phosphocholine (PC), glycerophosphoethanolamine (GPE), glycerophosphocholine (GPC), inorganic phosphate (Pi), phosphocreatine (PCr), and a-, b-, and g-adenosine triphosphate (ATP). The magnesium ( $\mathrm{Mg}^{2+}$ ) levels and pH were calculated based on PCr, Pi and b-ATP chemical shifts. Results: Compared to controls, the MCD lesions exhibited lower pH values and higher $\mathrm{Mg}^{2+}$ levels ( $p$ <0.05). The lesions also presented significant reduction of GPC and PDE, and an increased PME/PDE ratio. The otherwise normal appearing parenchyma also demonstrated lower $\mathrm{pH}$ values in the frontoparietal cortex and bilateral centrum semiovale. Conclusions: Our data support the idea that metabolic impairments occur in the lesions of MCD, with propagation to remote normal appearing parenchyma. The results also suggest that there are membrane turnover disturbances in MCD lesions.
\end{abstract}

Key words: metabolism, epilepsy, phospholipids, hydrogen-ion concentration, magnesium, cell membrane. 